\title{
Czochralski Growth of Decagonal AlCoNi Quasicrystals from Al-rich Solution
}

\author{
G. Meisterernst, B. Bauer and P. Gille* \\ Ludwig-Maximilians-Universität München, Department of Earth and Environmental Sciences \\ Crystallography section, Theresienstr. 41, D-80333 Munich, Germany
}

\begin{abstract}
Czochralski growth of $\mathrm{cm}$ size decagonal AlCoNi single crystals from Al-rich high-temperature solutions is described using native seeds oriented parallel to all symmetrically different crystal directions. Morphological observations allow first hints with respect to anisotropic growth rates. But only classical detachment experiments according to the original idea of Jan Czochralski reveal quantitative results of kinetically limited growth rates. Geometric conditions of wetted plane interfaces as well as aspects of constitutional supercooling affect the detachment experiments. Thus, in only one specific orientation of the decagonal quasicrystal quantitative data for the maximum growth rate could be obtained.
\end{abstract}

DOI: 10.12693/APhysPolA.124.344

PACS: 61.44.Br, 81.10.Aj

\section{Introduction}

With the discovery of quasicrystals in the binary Al-Mn system by Shechtman et al. [1] a new principle of arrangement of condensed matter was found. The studied phase showed icosahedral symmetry which is not compatible with three-dimensional translation periodicity in a conventional crystallographic understanding. Here the new ordering principle is quasiperiodicity which is characterised by long-range order but simultaneously missing periodicity. With the discovery of decagonal quasicrystals by Bendersky [2] it could be shown that the two contradictory principles of periodic and quasiperiodic ordering can be realised in just one solid at the same time.

Up to now, quite a few intermetallic systems exist in which thermodynamically stable decagonal quasicrystals occur. The decagonal phase in the $\mathrm{Al}-\mathrm{Co}-\mathrm{Ni}$ system has been studied most intensively and the crystals in this system are characterised best. These so called axial quasicrystals are geometrically built up of quasiperiodic layers which are stacked along a periodic axis. The description of these planes in a conventional crystallographic way leads to higher-dimensional space. The quasiperiodic planes can be regarded periodic in the $(4+1)$-dimensional space. Four reciprocal basis vectors $\boldsymbol{a}_{i}^{*}=a^{*}(\cos 2 \pi i / 5, \sin 2 \pi i / 5,0)(i=1,2,3,4)$ are needed in the quasiperiodic plane, while the fifth vector $\boldsymbol{a}_{5}^{*}$ is parallel to the stacking direction [3]. Therefore, five independent indices $\left\{h_{1} h_{2} h_{3} h_{4} h_{5}\right\}$ describe crystallographic forms of decagonal quasicrystals. The point group symmetry is $10 / \mathrm{mmm}$. The stereographic projection of the morphological forms, which we observed in several growth experiments of $\mathrm{AlCoNi}$ quasicrystals [4] is shown in Fig. 1.

*corresponding author; e-mail: gille@lmu.de

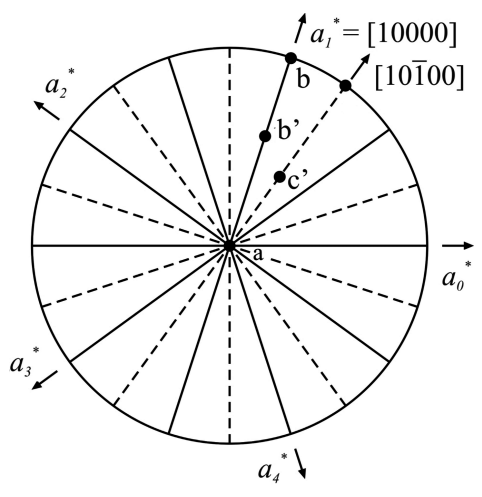

Fig. 1. Stereographic projection of the observed forms occurring in the crystal class $10 / \mathrm{mmm}$ : a $\{00001\}$ : pinacoid; b $\{10000\}$ : decagonal prism; $b^{\prime}\{0 \overline{1} \overline{1} 01\}$ : decagonal dipyramid; c $\{10 \overline{1} 00\}$ : decagonal prism; $c^{\prime}\{10 \overline{1} 02\}$ : decagonal dipyramid.

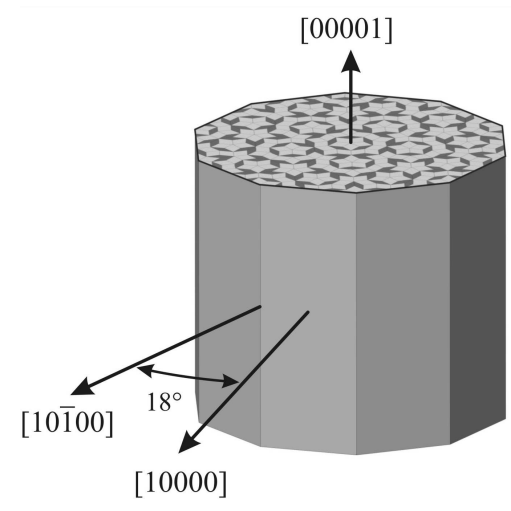

Fig. 2. The two symmetrically non-equivalent twofold axes $[10000]$ and $[10 \overline{1} 00]$ span the quasiperiodic layers, represented here by the famous Penrose tiling [5], which are stacked along the tenfold rotation axis [00001]. 
Figure 2 shows the two symmetrically non-equivalent directions [10000] and [10100] which span the quasiperiodic layer. The angle between these directions is $18^{\circ}$. They correlate with the two different twofold axes in this crystal class, while the tenfold rotation axis runs parallel to [00001], the stacking direction of the quasiperiodic planes. It is the aim of this paper to contribute to the understanding of anisotropic growth kinetics observed in crystal growth of decagonal AlCoNi quasicrystals using the Czochralski method from Al-rich solutions. For this aim, we describe detachment experiments to determine the maximum crystallization rates of crystallographic forms that could be identified during the Czochralski growth experiments [4].

\section{Czochralski growth of decagonal quasicrystals}

In the ternary $\mathrm{Al}-\mathrm{Co}-\mathrm{Ni}$ system, the decagonal phase melts peritectically, but can be grown directly from an Al-rich solution. In Fig. 3 the isothermal section of the Al-rich part of the $\mathrm{Al}-\mathrm{Co}-\mathrm{Ni}$ phase diagram at $1050^{\circ} \mathrm{C}$ [6] shows the two-phase coexistence region between the liquid (L) and the solid decagonal phase (D).

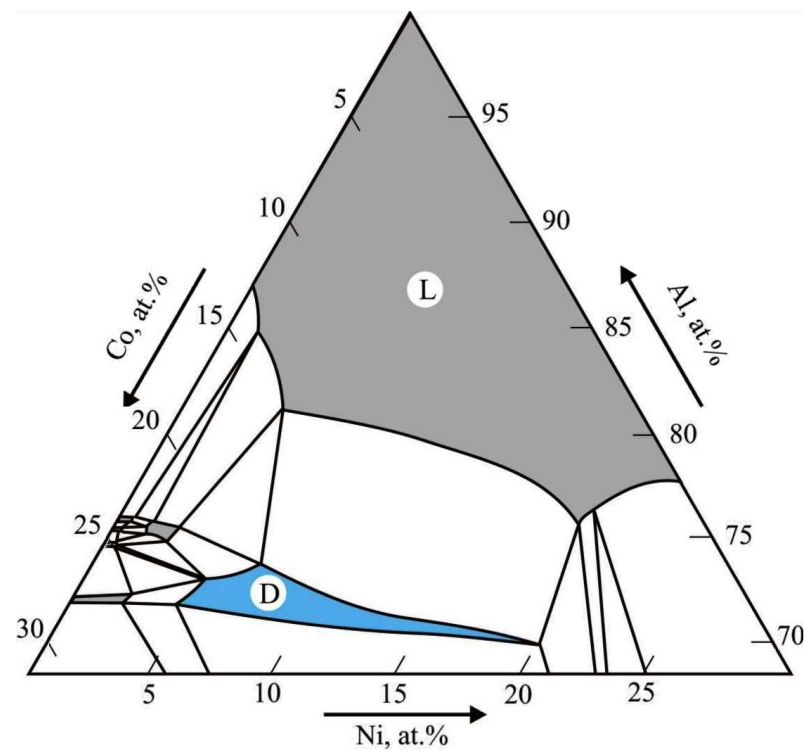

Fig. 3. Isothermal section of the Al-rich part of the $\mathrm{Al}-\mathrm{Co}-\mathrm{Ni}$ phase diagram at $1050{ }^{\circ} \mathrm{C}$, after [6].

According to this phase diagram the decagonal phase can only be grown from off-stoichiometric melts. The Czochralski method is the most favourable method to grow this kind of single crystals, because it allows perfect observation of the growth process. Large decagonal AlCoNi single crystals have been grown using different initial melt compositions. Exemplarily, crystals grown from the initial melt composition of $\mathrm{Al}_{77} \mathrm{Co}_{6} \mathrm{Ni}_{17}$ will be discussed further. The composition of crystals obtained from this melt was measured to be $\mathrm{Al}_{72} \mathrm{Co}_{9} \mathrm{Ni}_{19}$ using electron probe microanalysis.
Growing crystals from off-stoichiometric melts or rather Al-rich solutions involves some specifics that have to be considered. During growth the composition of the melt continuously changes depending on the amount of material already crystallized. Therefore, the liquidus temperature of the melt decreases permanently which has to be accommodated by using an appropriate temperature programme. More precisely, in the case of Al-rich solutions, rejected aluminium will be enriched in the melt, so the liquidus temperature will drop during growth. Accordingly, a progressively decreasing temperature programme in the range of $0.1-1 \mathrm{~K} / \mathrm{h}$ has to be applied to ensure a stable height of the meniscus and a constant diameter simultaneously. To ensure an adequate transport of the excess component away from the phase boundary good mixing of the melt and a low pulling velocity have to be guaranteed. For realisation, counter rotation of crystal and crucible cares for the good mixing of the melt and a pulling velocity of $0.15 \mathrm{~mm} / \mathrm{h}$ has found to be sufficient for the growth of decagonal $\mathrm{AlCoNi}$ quasicrystals. In addition to the good observation of the growth process which allows to check the crystal quality already at a very early stage, the possibility to grow crystals in different crystallographic orientations by using appropriate seeds is one of the main advantages of the Czochralski method.

\section{Anisotropy of growth rates and morphology}

From the Czochralski growth experiments of decagonal quasicrystals anisotropic growth rates of different crystallographic orientations could be observed. In case of choosing one of the symmetrically non-equivalent twofold rotational axes as pulling direction, the cross-section will be oval shaped with the longer extension parallel to the periodic direction [00001] (see Fig. 4a). Although the anisotropy is not directly measurable in a growth experiment, this is a strong hint that despite the forced rotationally symmetrical conditions within the melt the oval shape of the crystals is due to different growth velocities parallel and perpendicular to the periodic direction with the growth parallel to the periodic direction being faster. Quasicrystals pulled parallel to the tenfold rotational axis [00001] show a circular cross-section (see Fig. 4b). From this can be deduced that the two symmetrically non-equivalent twofold rotational axes [10000] and [10100], which are perpendicular to the tenfold axis, have similar crystallization rates. Using a pulling direction somewhat inclined to the tenfold rotational axis causes a change in shape of the cross-section of the quasicrystal as well. The contribution of the tenfold direction with its large growth rate causes the quasicrystal to extend into this direction, leading to a crystal that grows inclined to the pulling axis.

Growing crystals parallel to one of the symmetrically non-equivalent twofold axes results in different morphologies of the solid-liquid interface. Crystals grown parallel to the [10000] direction show a flat (10000) interface, while for crystals grown parallel to the other twofold 


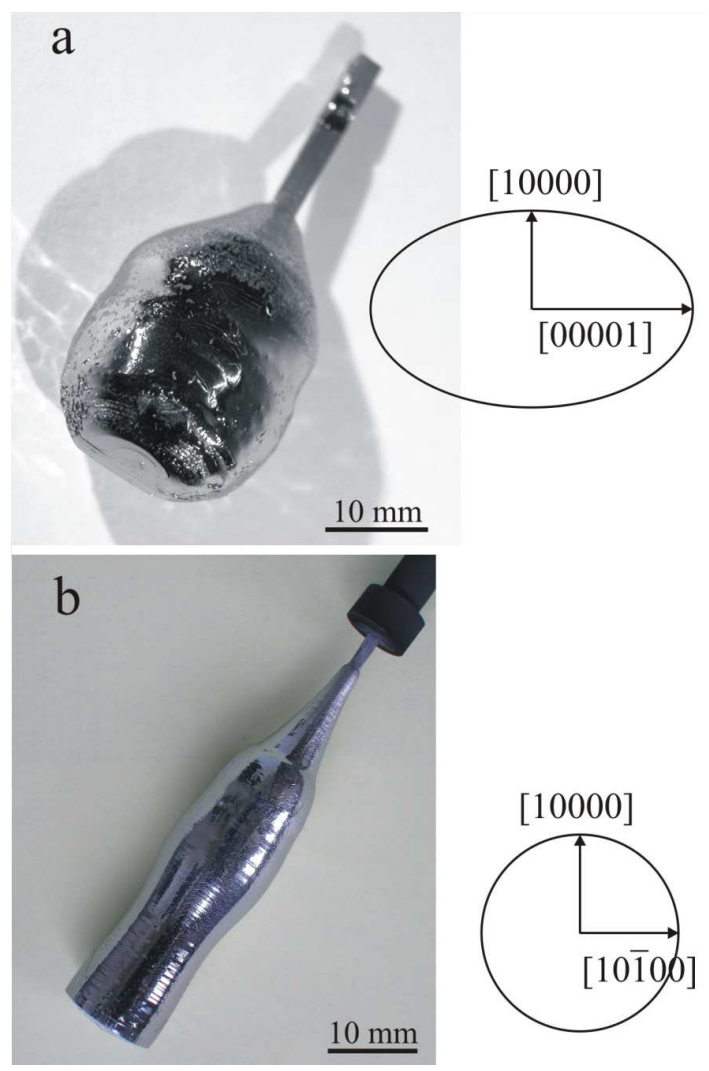

Fig. 4. (a) Crystal grown parallel to the aperiodic direction [10 $\overline{1} 00]$ and (b) along the periodic direction [00001] and the corresponding cross-sections, respectively.

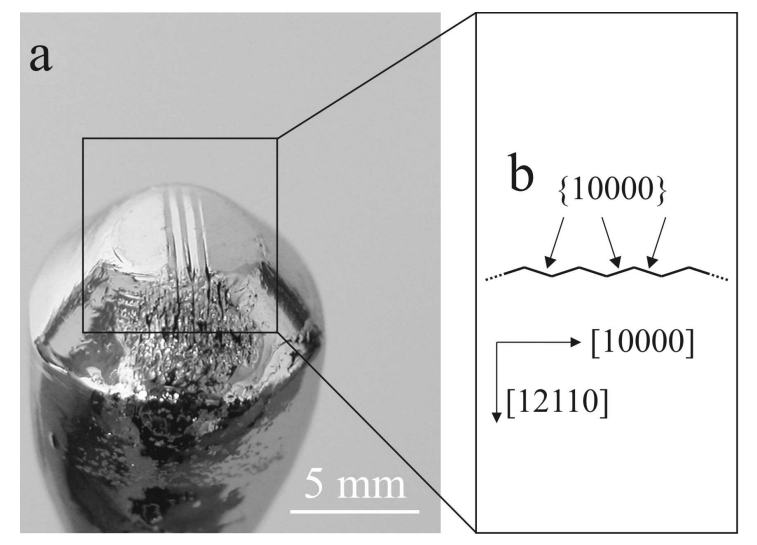

Fig. 5. (a) Interface of a crystal grown parallel [12110]; (b) sketch of the axial cut through the interface with facets of the form $\{10000\}$.

direction [10 $\overline{1} 00]$ the corresponding perpendicular plane does not develop. Here, the interface is made up of a sequence of $\{10000\}$ facets as shown in Fig. 5. For crystals grown along the periodic direction [00001] no interface faceting was observed and the interface follows a curved isotherm.

\section{Detachment experiments}

As mentioned before, it is the Czochralski method, which is most suitable concerning observability and control of the crystal growth process. Here it will be used in its original intent to determine the growth rate of different crystallographic orientations of decagonal quasicrystals. In his original paper [7], Czochralski suggested a method to determine the growth velocity of low-melting metals. This was done by pulling a thin single-crystalline metal fibre from the melt. When a pulling rate higher than the specific maximum crystallization velocity of the metal is applied, the crystal separates from the melt.

\subsection{Background of Czochralski's detachment experiment}

In Czochralski's original paper [7], it was already mentioned that the crystal-liquid interface is at a level well above the average melt surface and the crystal diameter is determined by the meniscus shape. The latter can be influenced by differences between the experimentally chosen pulling rate $v_{\mathrm{p}}$ and the axial growth rate $v_{\mathrm{g}}$ which is driven by thermal and kinetic conditions. Under equilibrium conditions (Fig. 6a) growth at constant crystal diameter occurs at $v_{\mathrm{g}}=v_{\mathrm{p}}$ while at a higher pulling rate $v_{\mathrm{p}}^{+}$the difference $\left(v_{\mathrm{p}}^{+}-v_{\mathrm{g}}\right)$ leads to a narrowing of the crystal diameter that is e.g. used in Dash's necking procedure [8] as to eliminate dislocations in Si crystal growth.

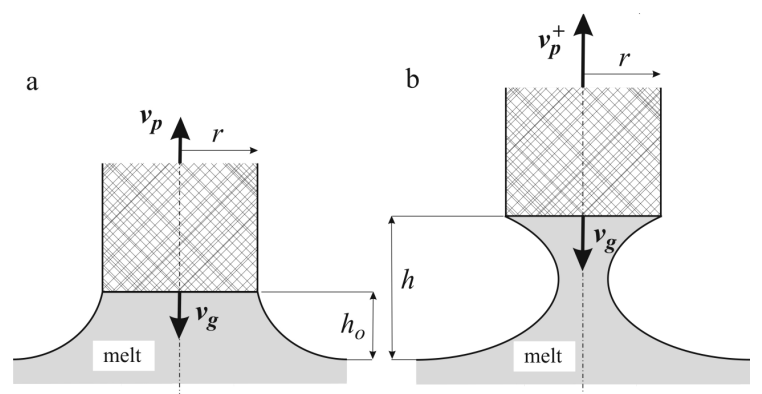

Fig. 6. Schematic sketch of the meniscus shape in Czochralski growth from the melt under steady-state conditions where the axial growth rate $v_{\mathrm{g}}$ equals the pulling rate $v_{\mathrm{p}}(\mathrm{a})$, and with an increased pulling rate $v_{\mathrm{p}}^{+}$that exceeds the maximum growth rate and ends with the detachment of the crystal from the melt (b).

Under equilibrium conditions, the height $h_{0}$ of the increased meniscus can be theoretically described, e.g. by Hurle's formula [9]. The question at which maximum meniscus height $h_{\max }$ the melt can no longer be held in contact with the crystal is simply a mechanical problem resulting from the balance of surface tension and gravitational forces.

Following Czochralski's original idea [7], we assume that a maximum growth rate $v_{\mathrm{g}, \max }$ exists for some reason, e.g. caused by kinetic constraints. With a constant pulling rate $v_{\mathrm{p}}^{+}$that exceeds this limit, the meniscus height increases with time $t$ to a position $h>h_{0}$ 
(Fig. 6b) and can be expressed by

$$
h=h_{0}+t\left(v_{\mathrm{p}}^{+}-v_{\mathrm{g}, \max }\right) .
$$

It reaches its maximum height $h_{\max }$ after applying the too high pulling rate during a time $t_{\max }$ and ends with the detachment of the melt from the growing crystal

$$
t_{\max }=\left(h_{\max }-h_{0}\right) /\left(v_{\mathrm{p}}^{+}-v_{\mathrm{g}, \max }\right) \text {. }
$$

Experimentally, the pulling rate in the Czochralski growth experiment described above has to be suddenly increased at $t=0$ to $v_{\mathrm{p}}^{+}$and held constant up to the moment $t_{\max }$ where the crystal loses contact to the melt. Using a series of different pulling rates all of them exceeding the maximum possible crystallization rate, Eq. (2) permits to calculate the maximum possible growth rate $v_{\mathrm{g}, \max }$ just from measuring the time $t_{\max }$ needed to detach the growing crystal from the melt. A linear plot of the reciprocal detachment time $\left(t_{\max }\right)^{-1}$ versus the various applied pulling rates $v_{\mathrm{p}}^{+}$gives the maximum growth rate by the extrapolation towards $\left(t_{\max }\right)^{-1}=0$.

\subsection{Quasicrystal detachment experiments}

To determine the maximum growth rates $v_{\mathrm{g} \text {,max }}$ of the decagonal quasicrystals along the $\left[h_{1} h_{2} h_{3} h_{4} h_{5}\right]$ growth direction, a series of experiments was performed. After seeding and stable growth of the crystal at constant diameter in the specific orientation, the pulling speed $v_{\mathrm{p}}$ was increased abruptly to a value $v_{\mathrm{p}}^{+}>v_{\mathrm{p}}$ and the time $t_{\max }$ between increase of the pulling speed and separation of the crystal from the melt was measured. By repeating this procedure with applying different pulling velocities $v_{\mathrm{p}}^{+}$a series of $t_{\max }\left(v_{\mathrm{p}}^{+}\right)$values could be obtained. The result of the analysis of these values is the maximum crystallization velocity $v_{\mathrm{p} \text {,max }}$ of the specific orientation $\left[h_{1} h_{2} h_{3} h_{4} h_{5}\right]$.

In the course of the experiment the crystal was detached several times from the melt after having grown the crystal at stable conditions for some time. Just when the pulling rate was increased to a higher value $v_{\mathrm{p}}^{+}$, the recording of the time until the detachment of the crystal from the melt was started. The time measurement was done by video monitoring the situation at the elevated meniscus with a resolution of one frame per minute. This resolution is precise enough compared to the typical duration $t_{\max }$ of one single detachment experiment which is in the range of several hours.

With the first separation of the crystal from the melt, the first $t_{\max }\left(v_{\mathrm{p}}^{+}\right)$pair of values was gained. By detaching the crystal at full diameter from the melt as well as by wetting the crystal again, the resulting thermal shock induces structural imperfections that could influence further growth and affect the determination of the maximum crystallization rate $v_{\mathrm{g}, \max }$. Therefore, in order to achieve a state of stable crystal growth at a constant diameter, the disordered part of the crystal was remelted completely prior to each new detachment experiment. Dissolving a length of about $5 \mathrm{~mm}$ has shown to be adequate to reach undisturbed single-crystalline regions. Beginning at this position, a new crystal growth run to achieve a state of constant diameter could be started.
To compensate the decreasing liquidus temperature during growth due to the enrichment of aluminium in the melt, an appropriate temperature programme was applied simultaneously to each growth or dissolution step. This ensures the continued growth at a constant diameter. After a period of stable growth at constant diameter at a pulling velocity of $v_{\mathrm{p}}=0.15 \mathrm{~mm} / \mathrm{h}$, the pulling rate was again increased to a value $v_{\mathrm{p}}^{+}>v_{\mathrm{p}}$ to gain the next $t_{\max }\left(v_{\mathrm{p}}^{+}\right)$pair of values. This procedure of dissolving, growing and detaching followed by another growth run was repeated for every single $t_{\max }\left(v_{\mathrm{p}}^{+}\right)$pair of values. Because of the low growth rates during normal growth at $v_{\mathrm{p}}$ and the additional time used for dissolving, the procedure of the detachment experiments took about eight weeks for each crystallographic direction $\left[h_{1} h_{2} h_{3} h_{4} h_{5}\right]$. An absolute requirement for the feasibility of these detachment experiments were the stable conditions in the Czochralski setup. To prevent the growth atmosphere against oxygen the growth chamber is fully metal-sealed.

In decagonal quasicrystals three types of crystallographic directions differ qualitatively:

i) orientation parallel to the tenfold axis [00001]; perpendicular to the pinacoid,

ii) orientation parallel to one of the two symmetrically non-equivalent twofold axes $\langle 10000\rangle$ and $\langle 10 \overline{1} 00\rangle$; perpendicular to the faces of the two different decagonal prisms, and

iii) orientation parallel to a so-called inclined direction $\left\langle h_{1} h_{2} h_{3} h_{4} h_{5}\right\rangle$, where at least one of the indices $h_{1}$, $h_{2}, h_{3}$, or $h_{4} \neq 0$ and $h_{5} \neq 0$; with faces of a decagonal dipyramid perpendicular; in other words: any crystallographic direction showing a periodic and an aperiodic component.

The qualitative difference of these types of orientations expresses itself in the growth morphology of crystals grown parallel to these directions. Because of the known anisotropy of growth rates, as described before (see Sect. 3), the crystal cross-section at the liquid-solid interface is strongly influenced by the maximum growth velocities $v_{\mathrm{g}, \max }$ of the different crystallographic orientations $\left\langle h_{1} h_{2} h_{3} h_{4} h_{5}\right\rangle$.

\section{Results of detachment experiments 5.1. Parallel to the tenfold [00001] and the inclined directions}

After successful seeding parallel [00001], conditions for a constant diameter growth at $v_{\mathrm{p}}=0.15 \mathrm{~mm} / \mathrm{h}$ was set. On applying a higher pulling rate $v_{\mathrm{p}}^{+}$the crystal did not detach from the melt even after some hours of continued growth. Also by repeated increase of the pulling rate to values $v_{\mathrm{p}}^{+} \gg v_{\mathrm{p}}$ it was not possible to separate the crystal from the melt. Instead, the loss of the cylindrical shape of the growing crystal was observed. Detaching this crystal was only possible by a dramatic increase of the pulling rate to $v_{\mathrm{p}}^{+}=36 \mathrm{~mm} / \mathrm{h}$. The investigation of this crystal 
has shown that the liquid-solid interface decomposes into separated decagonal needles with residual Al-rich melt in between [10]. This structural decomposition of the growth interface can be explained by the effect of constitutional supercooling [10]. Single-crystalline growth parallel to [00001] at pulling rates close to detachment conditions is not possible and therefore the maximum growth rate $v_{\mathrm{g}, \max }$ parallel to the tenfold axis cannot be determined. From this result it is obvious that it will not be possible to determine the maximum growth rate $v_{\mathrm{g} \text {,max }}$ of any crystallographic orientation of a decagonal quasicrystal that includes a component of the periodic direction [00001]. This leads to an exclusion of any inclined direction from the detachment experiments.

\subsection{Parallel to the twofold directions [10000]
and [10100]}

While pulling a crystal parallel to one of the two crystallographically non-equivalent twofold directions [10000] and [10100] no component of the tenfold direction [00001] is parallel to the pulling direction. As known from previous growth experiments, the liquid-solid interface for crystals grown parallel to the twofold directions [10000] and [10100] is formed by crystallographic faces of the decagonal prism $\{10000\}$ whereas the decagonal prism $\{10 \overline{1} 00\}$ has no morphological relevance as a growth face. The pulling speed used for constant diameter growth was $0.15 \mathrm{~mm} / \mathrm{h}$ for both directions. During the detachment experiment by choosing the twofold direction [10 $\overline{100}$ ] as pulling direction, the crystal was eight times grown and separated from the melt. The $t_{\max }\left(v_{\mathrm{p}}^{+}\right)$data so obtained show a linear dependence as shown in Fig. 7.

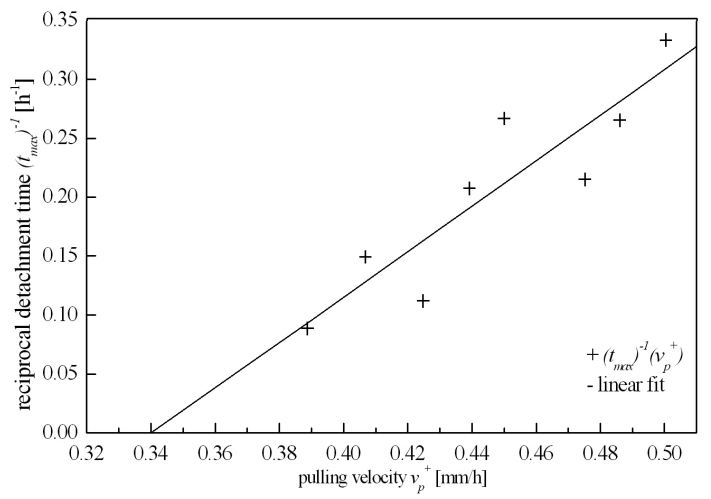

Fig. 7. Linear plot of $\left(t_{\max }\right)^{-1}$ vs. $v_{\mathrm{p}}^{+}$for pulling parallel to $[10 \overline{1} 00]$.

Using a simple linear regression (see Eq. (3)) the maximum growth rate $v_{\mathrm{g} \text {,max }}$ for the growth parallel to the [10100] direction was found at the intersection for the reciprocal detachment time $\left(t_{\max }\right)^{-1}=0$ :

$$
\left(t_{\max }\right)^{-1}=(-0.65 \pm 0.17)+(1.9 \pm 0.4) \times v_{\mathrm{p}}^{+} .
$$

Accordingly, the maximum growth rate $v_{\mathrm{g}, \max }$ for the [10100] direction amounts to $(0.34 \pm 0.04) \mathrm{mm} / \mathrm{h}$.

The situation changes by performing the experiment parallel to the second twofold axis [10000] as the pulling direction. In this case, the process of growing, detaching, and dissolving the crystal has been repeated nine times in the same range of pulling rates $v_{\mathrm{p}}^{+}$. But in contrast to the experiment parallel to the other twofold direction a much higher scattering of data was obtained making a linear regression not meaningful. Since in both cases the liquid-solid interface is formed by large facets of the crystallographic form $\{10000\}$, the only reason for this different behaviour could be the different orientation of the interface with respect to the pulling direction and the melt surface. In the case of pulling parallel to the [10000] direction the growth front is formed by a single (10000) facet that is perpendicular to the growth direction and parallel to the melt surface. In contrast, the growth front in case of pulling parallel to the [10100] direction is formed by a set of $\{10000\}$ facets that are inclined by $18^{\circ}$ with respect to the melt surface (see Fig. 5). We regard this to be a simple geometrical problem instead of something that is driven by the growth kinetics, since the growth interface is the same one in both cases.

To investigate the influence of these different geometries at the two-phase boundary a model experiment was built up. Two simple test species of the same diameter made of stainless steel have been shaped in order to represent the two interfaces under investigation. For these two interface models detachment experiments were performed using water as a model melt and the maximum possible elevation of the water meniscus after wetting was determined. With wetting and detaching each model interface many times, the repeatability for each type of interface could be determined. As a result, the water meniscus at that model representing the complex interface perpendicular to the [10 $\overline{1} 00]$ direction with inclined faces could be elevated to its maximum height much more precisely than with the model representing the singular flat interface (10000) perpendicular to the vector of gravity showing the same data scattering as in the detachment experiments with the quasicrystal described above.

With this, the difference in the accuracy of the results for the maximum growth rates $v_{\mathrm{g} \text {,max }}$ of the two different twofold directions [10000] and [10100] could be better understood. But, we end up with the somehow disappointing result that only one of all tested growth directions allowed a quantitative analysis of the maximum possible growth rate.

\section{Summary}

The Czochralski method has proved to be a suitable technique to grow decagonal AlCoNi crystals from an Al-rich solution at temperatures of about $1050^{\circ} \mathrm{C}$. Highly perfect single crystals were grown from native seeds by pulling either parallel to the tenfold axis [00001] which is the periodic direction of decagonal quasicrystals or along one of the crystallographically non-equilvalent twofold rotational axes [10000] and [10̄100] having quasiperiodic order. Hints of anisotropic growth can already be obtained from the cross-section of decagonal quasicrystals that has 
an elliptical shape with the larger extension along the periodic [00001] direction.

In order to investigate the growth anisotropy quantitatively we adopted the original idea of Jan Czochralski and used his method to measure the maximum possible growth rate along all qualitatively different directions of the decagonal AlCoNi quasicrystal. Since crystal growth parallel to the periodic axis is very fast, kinetic limits of growth could not be found prior to the onset of constitutional supercooling. This is also true for pulling along any direction that includes a component of the periodic axis, i.e. parallel to so-called inclined directions.

The Czochralski growth parallel to one of the twofold axes is kinetically limited allowing the classical detachment experiments when pulling at a higher rate than the maximum possible growth rate. But, geometric reasons depending on the orientation of the plane interfaces with respect to the vector of gravity determine whether or not the detachment experiments give clear results. Pulling decagonal AlCoNi quasicrystals parallel to the [10100] axis has resulted in a kinetically limited maximum growth rate of $(0.34 \pm 0.04) \mathrm{mm} / \mathrm{h}$.

\section{Acknowledgments}

The work has been supported by the Deutsche Forschungsgemeinschaft under contract Gi 211/5.

\section{References}

[1] D. Shechtman, I. Blech, D. Gratias, J.W. Cahn, Phys. Rev. Lett. 53, 1951 (1984).

[2] L. Bendersky, Phys. Rev. Lett. 55, 1461 (1985).

[3] W. Steurer, T. Haibach, in: Physical Properties of Quasicrystals, Ed. Z.M. Stadnik, Springer Series in Solid-State Sciences, Vol. 126, Springer-Verlag, Berlin 1999.

[4] P. Gille, G. Meisterernst, N. Faber, J. Cryst. Growth 275, 224 (2005).

[5] R. Penrose, Bull. Inst. Math. Appl. 10, 266 (1974).

[6] T. Gödecke, M. Scheffer, R. Lück, S. Ritsch, C. Beeli, Z. Metall. 89, 687 (1998).

[7] J. Czochralski, Z. Phys. Chem. 92, 219 (1918).

[8] W.C. Dash, J. Appl. Phys. 30, 459 (1959).

[9] D.T.J. Hurle, B. Cockayne, in: Handbook of Crystal Growth. Bulk Crystal Growth, Vol. 2a, Ed. D.T.J. Hurle, North-Holland, Amsterdam 1994, p. 99.

[10] G. Meisterernst, Ph.D. Thesis, Ludwig-MaximiliansUniversität München, Munich 2006. 